\title{
Estado de ánimo y memoria autobiográfica: efectos sobre la intensidad de los recuerdos escolares
}

\author{
José T. Boyano* y Juan A. Mora
}

Departamento de Psicología Básica. Facultad de Psicologia. Universidad de Málaga. (España).

\begin{abstract}
Resumen: La influencia del estado de ánimo sobre la memoria de estímulos simples ha sido constatada con frecuencia. El presente trabajo examina el efecto del ánimo sobre la memoria autobiográfica, dirigiéndose a analizar (1) la presencia del efecto de memoria congruente con el ánimo en los recuerdos escolares y (2) las anomalías típicas del efecto. Se pretende investigar los sesgos que pueden afectar, durante la recuperación, a la valoración subjetiva de eventos relevantes de la historia escolar. Se ha utilizado una muestra de estudiantes universitarios, asignados a distintos grupos de in ducción de ánimo. Se han inducido tres estados (alegría, tristeza o ánimo neutro) mediante material fílmico que presenta escenas emocionales. Se registró la intensidad atribuida al recuerdo mediante el método LIM. El efecto de memoria congruente con el ánimo ha sido parcial y asimétrico. Nuestros resultados indican que los participantes con ánimo alegre tienden a una mayor valoración de los eventos positivos y negativos, mientras las personas con tristeza tienden a valoraciones más conservadoras. Los participantes con ánimo neutro valoran los eventos positivos con un nivel más elevado que los negativos. Estos datos sugieren que el ánimo genera un estilo de procesamiento preferente que modula la valoración de los recuerdos.

Palabras clave: Memoria autobiográfica; ánimo; intensidad; memoria congruente con ánimo; historia escolar.
\end{abstract}

\section{Introducción}

La relación entre emoción y cognición, objeto de interés a lo largo de toda la historia de la psicología, constituye hoy un campo de investigación creciente. En concreto, la influencia de los estados afectivos sobre la memoria ha sido explorada desde distintas perspectivas. Para Tulving (1989), cuando la información procedente de la memoria es recuperada se actualiza en función de diversos factores, como la interpretación y comprensión de la experiencia pasada, las nuevas informaciones y los estados actuales del individuo. En esta línea, la influencia del humor sobre la recuperación selectiva de recuerdos congruentes ha sido un fenómeno reproducido con frecuencia en tareas de laboratorio que involucran la memoria explícita, como el aprendizaje de palabras mediante recuerdo libre (Ellis y Moore, 1999; Fernández-Castro, Granero, Barrantes y Capdevila, 1997; Singer y Salovey, 1988) o reconocimiento (Meilán et al., 2012), así como memoria implícita (Barry, Naus y Rehm, 2004).

En el campo de la memoria autobiográfica también se encuentran numerosas evidencias del fenómeno de memoria congruente con el ánimo (mood-congruent memory o MCM) utilizando distintas técnicas de inducción del ánimo (Bower, 1981; Christianson, 1992; Garcez y Cláudio, 2009; Miranda y Kihlstrom, 2005; Singer y Salovey, 1988). La mayor facilidad para recuperar recuerdos congruentes con el estado de ánimo constituye un fenómeno muy robusto (véase para revi-

* Dirección para correspondencia [Correspondence address]:

Jose T. Boyano. Facultad de Psicología. Universidad de Málaga. (España). Email: jose_boyano@uma.es

\begin{abstract}
Title: Mood and autobiographical memory: effects on the intensity of school memories.

Abstract: The influence of mood on memory for simple stimuli has been observed frequently. This paper examines the effect of mood on autobiographical memory, going to analyze (1) the presence of the moodcongruent memory effect in school memories and (2) the typical anomalies of the effect. We intend to study the biases that may affect, during retrieval, the subjective rating of relevant events in school history. We used a sample of college students assigned to different groups of mood induction. Three states (happy, sad or neutral mood) were induced by film material having emotional scenes. Memories intensity was recorded by the LIM method. The mood-congruent memory effect has been partial and asymmetric. Our results indicate that participants with happy mood tend to a greater rating of positive and negative events, whereas sad participants tend to more conservative valuations. Neutral-mood participants rated the positive events with a higher level than negative. These data suggest that the mood generates a preferred processing style which modulates the valuation of memories.

Key words: Autobiographical memory; mood; intensity; mood-congruent memory; school history.
\end{abstract}

sión, Holland y Kensinguer, 2010; Ellis y Ashbrook, 1991; Ellis y Moore, 1999; Matt, Vázquez y Campbell, 1992, para un meta-análisis), en cuanto a tamaño del efecto, consistencia y diversidad (Blaney, 1986). El efecto de congruencia se manifiesta en diversas medidas de la memoria autobiográfica: a) en la accesibilidad, o número de recuerdos recuperados; b) en la valoración de su valencia, o grado de positividad o negatividad; y c) en la latencia de respuesta, o tiempo que tardan los sujetos en recuperar recuerdos de una valencia determinada.

La congruencia con el estado de ánimo es evidente en el número de recuerdos positivos y negativos reportados después de una inducción del estado de ánimo, con un mayor número de recuerdos positivos recuperados tras una inducción positiva (Snyder y White, 1982). Estos investigadores analizaron la eficacia de la manipulación experimental: si las inducciones del ánimo no eran efectivas para generar el estado de ánimo, no se manifestaban efectos de memoria congruente. Es decir, las diferencias valorativas requerían que, en el momento del recuerdo de estos eventos, los participantes experimentasen en realidad los estados de ánimo de euforia o depresión. Normalmente, pues, la variable dependiente ha sido la valencia de los recuerdos recuperados, en recuerdo libre. Los individuos seleccionan diferentes tipos de recuerdos en función de la situación anímica (AlonsoQuecuty, 1990; Mathews y Bradley, 1983; Snyder y White, 1982). Por ejemplo, los sujetos bajo una inducción de ánimo alegre recuperan mayor número de recuerdos positivos. Además, después de una inducción del estado de ánimo feliz, los recuerdos son valorados como más positivos, comparados con grupos en los que se realiza una inducción de es- 
tado de ánimo triste (Madigan y Bollenbach, 1982; Mathews y Bradley, 1983), y los recuerdos positivos son percibidos como más cercanos (Gebauer, Broemer, Haddock y von Hecker, 2008). En conjunto, estos estudios han proporcionado resultados convergentes con el modelo de Red Asociativa del Afecto (Bower, 1981), pues se ha constatado de forma consistente que distintos estados anímicos pueden conducir a recuperar distintos contenidos.

Bower (1987) ha propuesto que el tema específico sobre el que se focaliza el estado de ánimo puede ser un factor importante para determinar si se producen efectos de congruencia. Esta visión concuerda con el modelo Self Memory System (SMS) de Conway y Pleydell-Pearce (2000), que se ha convertido en el modelo de referencia en el ámbito de la memoria autobiográfica. Conway y Pleydell-Pearce proponen que la información autobiográfica está organizada de forma jerárquica, con información conceptual o abstracta sobre uno mismo en los niveles superiores; información genérica acerca de eventos generales, repetidos o resumidos en los niveles intermedios; $y$, finalmente, información más específica en los niveles inferiores. La memoria autobiográfica sería una red flexible, con múltiples vías de acceso a diferentes informaciones. El principio estructurador se basaría en determinados temas que son especialmente relevantes para el individuo (trabajo, relaciones...) o metas activas. Así, en función de estas metas activas se produciría el acceso flexible -tanto en sentido ascendente como descendente- a la información autobiográfica.

En suma, el modelo SMS asume que la situación actual del individuo puede afectar a los procesos de recuperación autobiográfica, facilitando determinados contenidos e inhibiendo otros. Además, no se excluye que los contenidos puedan ser modificados con el paso del tiempo, como consecuencia de cambios en la vida personal. Los estudios dirigidos a constatar sesgos reconstructivos en la memoria emocional generalmente han constatado cambios valorativos que se producen después de periodos largos de tiempo, en términos de meses o años, y que afectan a un evento diana determinado (Levine, 1997; Levine y Bluck, 1997, 2004). Estas aproximaciones suponen que la nueva información sobre un tema puede afectar a las valoraciones de eventos pasados, asumiendo la metáfora de mapas que son actualizados.

En líneas generales, el efecto MCM puede ser explicado por los modelos que describen el funcionamiento de la memoria personal. No obstante, hay que señalar que se han encontrado fallos en la obtención del efecto MCM en determinados estudios (Blaney, 1986; Hasher et al., 1985). Además, se ha observado una asimetría positiva en el efecto de congruencia (Blasco et al., 1997; Isen, 1985), ya que se ha detectado mayor facilidad para reproducir el efecto en estados de ánimo positivos. Por ejemplo, Blasco, Borràs, Rey, Bonillo y Fernández-Castro (1997), utilizando películas, encontraron efectos de memoria congruente con el ánimo sólo tras la inducción de ánimo positivo. La conclusión final de Blasco et al. (1997) indica que el procedimiento de inducción provoca cambios significativos en las medidas de tono afectivo y produce un efecto de sesgo en el recuerdo en los casos de inducción positiva.

La memoria autobiográfica juega un papel relevante en la identidad y la regulación del ánimo en la adolescencia y la juventud. Por este motivo, el presente trabajo se dirige a explorar dos cuestiones importantes sobre la recuperación autobiográfica: (1) la influencia del efecto MCM en los recuerdos de la vida escolar; y (2) las anomalías y asimetrías típicas del efecto. Con este doble objetivo, se ha adaptado el paradigma MCM, igualando el número de eventos positivos y negativos, y registrando como variable dependiente la intensidad emocional atribuida a los recuerdos.

En primer lugar, se pretende contrastar si el estado de ánimo actual afecta a los procesos de recuperación de contenidos autobiográficos, modulando la reconstrucción mnésica de eventos de la historia personal pertenecientes al ámbito escolar. En este marco experimental, el modelo de la Red Asociativa del Afecto de Bower (1981) supone que la activación se propaga desde los nodos emocionales hacia otros contenidos de la misma valencia afectiva. Podemos suponer que los efectos asociativos facilitarán la recuperación de contenidos congruentes, y trataremos de comprobar si se producen sesgos en la reconstrucción de recuerdos emocionales con gran significación personal, incorporados a la historia vital. Es decir, buscaremos reproducir el efecto MCM en el ámbito de los recuerdos escolares, intentando incrementar la validez ecológica. Para ello, en el diseño experimental se incluirán distintos vídeos de inducción del ánimo que reproducirán situaciones naturales, relativas a distintos contextos, educativos o más generales.

En segundo lugar, intentaremos explorar si se producen las inconsistencias y asimetrías observadas en algunas investigaciones y determinar si estas asimetrías en el efecto MCM pueden ser explicadas por los modelos teóricos existentes.

\section{Método}

\section{Participantes}

La muestra está formada por 569 estudiantes de primer curso de Psicología (65.89\% de mujeres y 34.11\% de varones), asignados aleatoriamente a los grupos. Se obtuvo una edad media de 21.89 años $(D T=2.53)$ para los varones y 20.74 años $(D T=1.67)$ para las mujeres, con un rango entre 18 y 40 años.

\section{Material e Instrumentos}

El material elegido para generar el estado afectivo se basa en estímulos fílmicos. El fragmento debe provocar reacciones muy similares en la mayoría de los participantes, evitando respuestas emocionales muy fuertes, que implicarían mayor variabilidad intersujeto (McHugo, Smith y Lanzetta, 1982). El rango de duración de los clips está comprendido entre 4 minutos y 28 segundos y 5 minutos y 7 segundos. 
El material fílmico es elegido para inducir tres estados afectivos: alegría, tristeza o ánimo neutro. Cada emoción es presentada en un contexto general o en un contexto académico: 1) Alegría-Contexto general (Duración: 4' 30'). Escena familiar, con bebés que emiten risas. 2) TristezaContexto general. Fragmento de la película The Champ (4' 47'), utilizado para elicitar sentimientos de valencia negativa (Blanchard, Bellack y Mueser, 1994; Fernández Berrocal y Extremera, 2006; Macht, Roth y Ellgring, 2002; Jakobs, Manstead y Fischer, 2001). 3) Ánimo neutro-Contexto general (Duración: 4' 30'). Escena natural del fondo marino (Kreibig, 2004). 4) Alegría-Contexto académico (4'28'). Un grupo de estudiantes expresan alegría mediante abrazos. 5) Tristeza-Contexto académico (5'07' $)$. Escenas de acoso escolar, seguidas de noticias de la televisión española sobre acoso en enseñanza media y universitaria. 6) Ánimo neutroContexto académico (5'). Información institucional sobre los estudios universitarios.

Para comprobar la eficacia de la inducción del ánimo se ha utilizado la Escala PANAS (Positive and Negative Affect Schedule, 1988) de Watson, Clark y Tellegen. Con la finalidad de disminuir la fatiga, se han seleccionado diez preguntas relevantes para la investigación, cinco ítems positivos y cinco negativos. Se han seguido los criterios de adaptación propuestos por Talarico, LaBar y Rubin (2004), utilizando una escala de tipo likert que va desde el nivel 1 hasta el nivel 7 (emoción intensa).
Para favorecer la espontaneidad y facilitar la recuperación se ha aplicado el método LIM (Schroots, 1996; Schroots y Assink, 2005), que permite recuperar recuerdos positivos o negativos pertenecientes a las distintas etapas educativas que conforman la historia escolar. Mediante una escala de tipo likert se registran las valoraciones de los participantes sobre la intensidad de los recuerdos recuperados (véase Rubin, Burt y Fifield, 2003; Talarico et al., 2004).

\section{Procedimiento}

Fase 1. Inducción de Ánimo (8 minutos). Se ha solicitado a los participantes que se concentren en las emociones que sienten mientras observan el fragmento de vídeo. Inmediatamente después, se pidió a los participantes que cumplimentasen la escala de afecto positivo y negativo (adaptación de la escala PANAS).

Fase 2. Tarea de Recuperación Autobiográfica (25 minutos). Siguiendo el método LIM (Schroots y Assink, 2005) se ha solicitado a los participantes generar seis recuerdos relevantes de la vida escolar, relacionados con tres eventos positivos y tres eventos negativos. A continuación, los participantes informan sobre la intensidad emocional que atribuyen al evento que dio origen al recuerdo. Exclusivamente para los grupos de tristeza, se presenta material fílmico de valencia positiva durante cinco minutos para evitar consecuencias indeseadas o malestar asociado a la inducción (Figura 1).

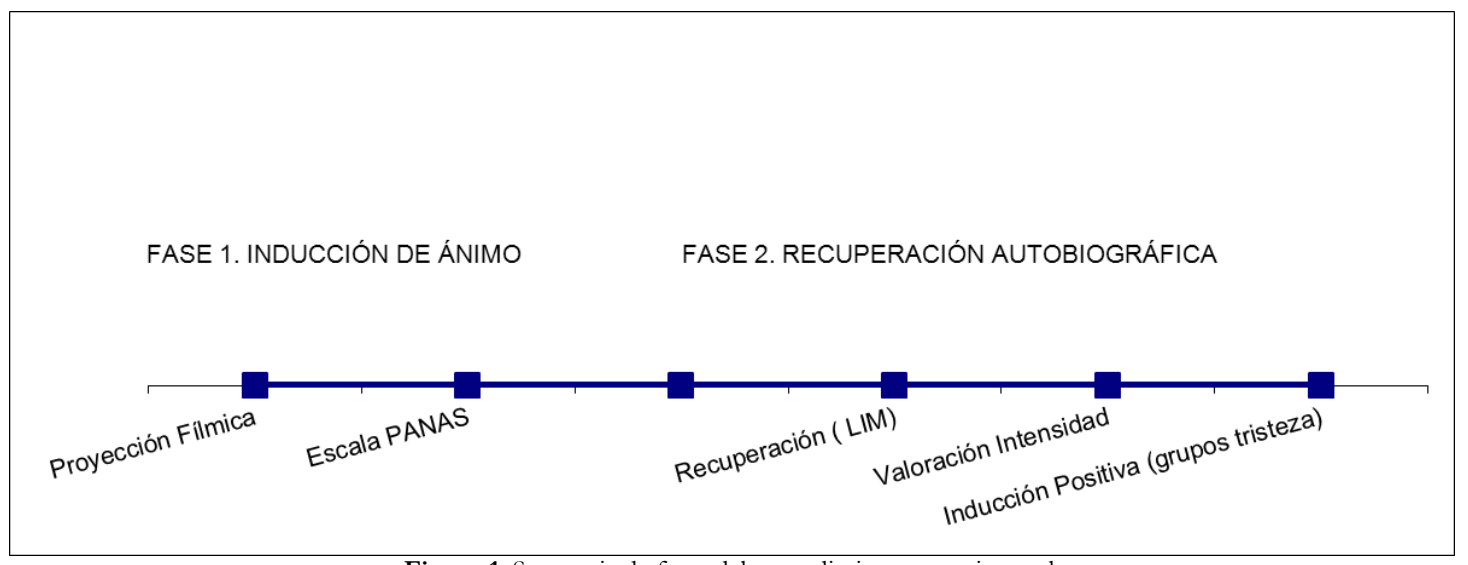

Figura 1. Secuencia de fases del procedimiento experimental.

\section{Resultados}

\section{Inducción del estado de ánimo}

Con el fin de comprobar la eficacia del procedimiento de inducción afectiva, se ha realizado un ANOVA unifactorial para el factor estado de ánimo inducido. Las medias obtenidas para la emoción objetivo en cada condición muestran una eficaz inducción del ánimo, como puede verse en la Figura 2. En la media que refleja el estado de alegría se obser- van diferencias significativas $\left[F(2.56)=785.74\right.$ y $p<.001, \eta^{2}$ $=.73$ entre los sujetos con estado de alegría inducida $(M=$ $6,21, D T=1.04)$, tristeza inducida $(M=1.49, D T=0.96) \mathrm{y}$ estado de ánimo neutro $(M=3.34, D T=1.49)$. En las medias obtenidas en el ítem que mide la tristeza se han encontrado también diferencias significativas $[F(2.56)=665.57 \mathrm{y}$ $\left.p<.001, \eta^{2}=.046\right]$ entre los grupos de alegría $(M=1.33, D T$ $=0.80)$, tristeza $(M=4.77, D T=1.64)$ y estado neutro $(M=$ $1.14, D T=0.46)$. 


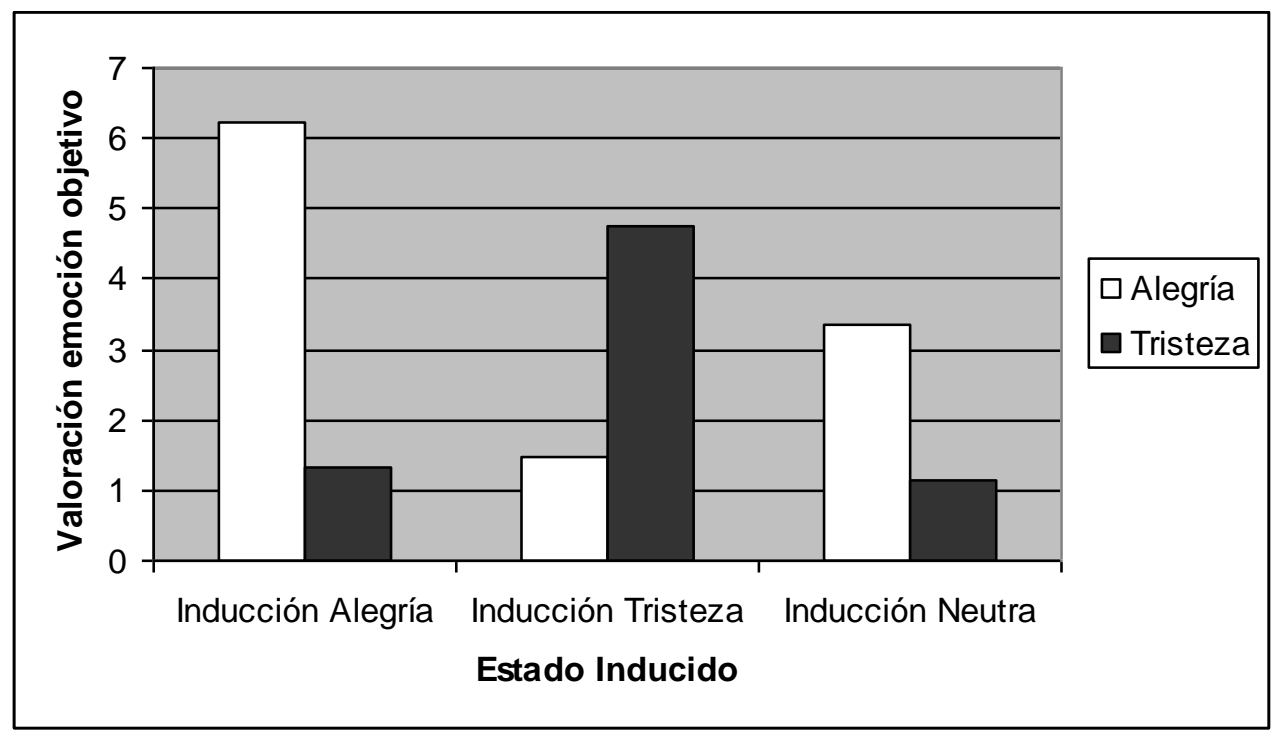

Figura 2. Medias de valoración de la emoción objetivo en función del estado de ánimo inducido.

\section{Valoración de la intensidad emocional del recuerdo}

Para comprobar la influencia del estado de ánimo sobre la recuperación, se ha realizado un ANOVA factorial $2 \times 3 \times$ 2 , con medidas repetidas en el factor valencia del evento, con el contexto situacional y el estado de ánimo inducido como factores intersujeto y la valencia del evento como factor intrasujeto. La variable dependiente medida ha sido la intensidad emocional atribuida a cada recuerdo. No se han encon- trado diferencias estadísticamente significativas en función del factor contexto situacional $[F(1.56)=0.41$ y $p=.520]$. Los participantes han valorado los eventos recordados de forma similar, tanto en las condiciones en que el contexto era académico como en las condiciones en que el film emocional se presentaba en un contexto general, como puede verse en la Figura 3. Dado que el contexto no parece tener una influencia significativa, en adelante nos centraremos en el análisis de resultados del factor estado de ánimo.

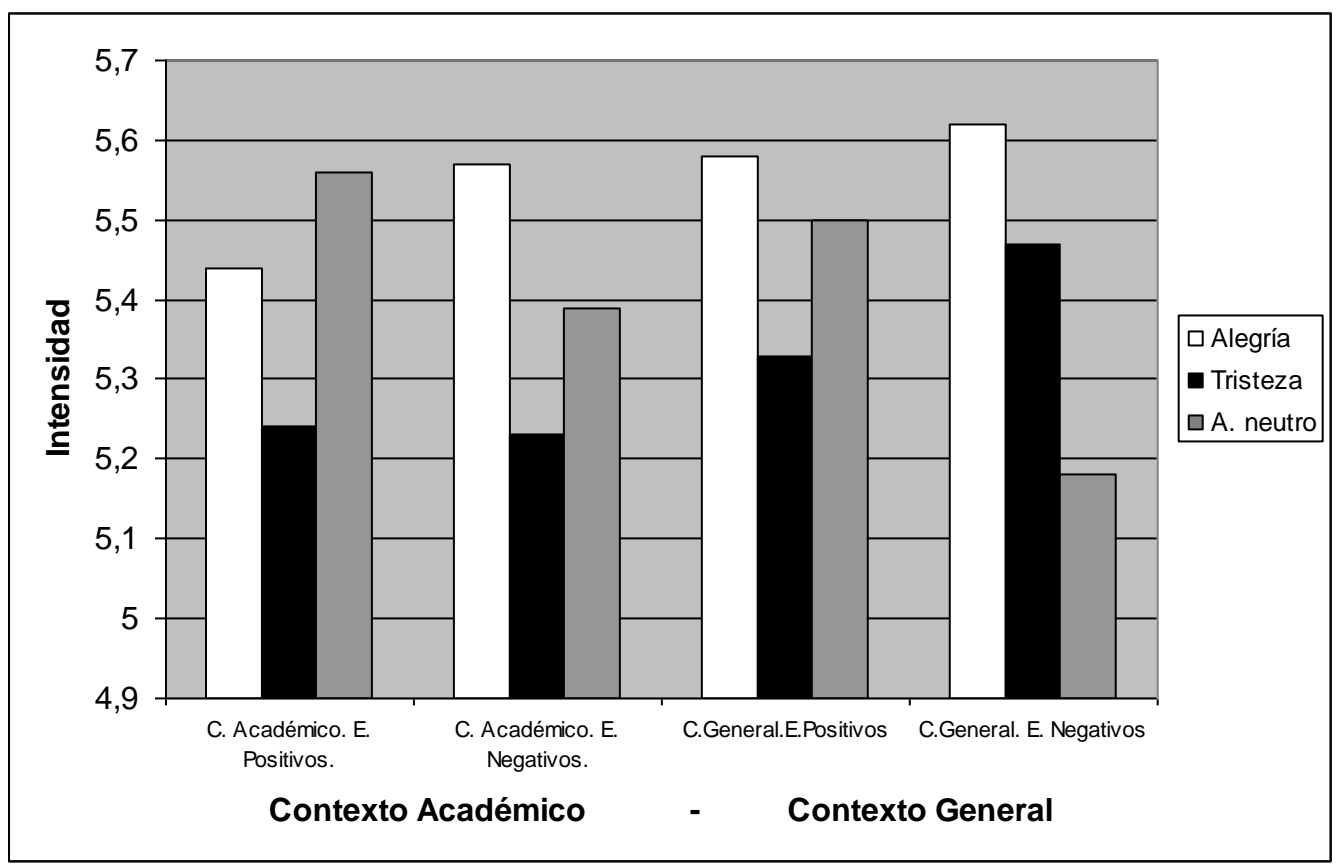

Figura 3. Medias de valoración de la intensidad en función del contexto, la valencia del evento y el estado de ánimo inducido (Alegría-Tristeza-A.Neutro). 
Los resultados muestran efectos significativos del estado de ánimo $\left[F(2.6)=4.5\right.$ y $\left.p=.09, \eta^{2}=.02\right]$. Los sujetos con estado de alegría han valorado los eventos con mayor intensidad emocional que los sujetos con tristeza $(M=5.5$ vs. $M$ $=5.31)$. La prueba de Scheffé muestra diferencias significativas entre ambas medias.

La interacción entre el estado de ánimo y la valencia del evento ha producido un efecto significativo $[F(2.6)=5.0 \mathrm{y} p$ $\left.=.06, \eta^{2}=.02\right]$. La interacción entre estado de ánimo y va- lencia del evento indica que cada estado de ánimo tiene un efecto distinto sobre la valoración de los planos positivo o negativo. De esta forma, cuando se ha inducido alegría todos los eventos se valoran con nivel elevado de intensidad; cuando se induce tristeza se valoran con nivel reducido; pero, críticamente, cuando el estado de ánimo es neutro, los eventos positivos se valoran con nivel elevado de intensidad, mientras los negativos se valoran a un nivel más reducido como puede verse en la Figura 4.

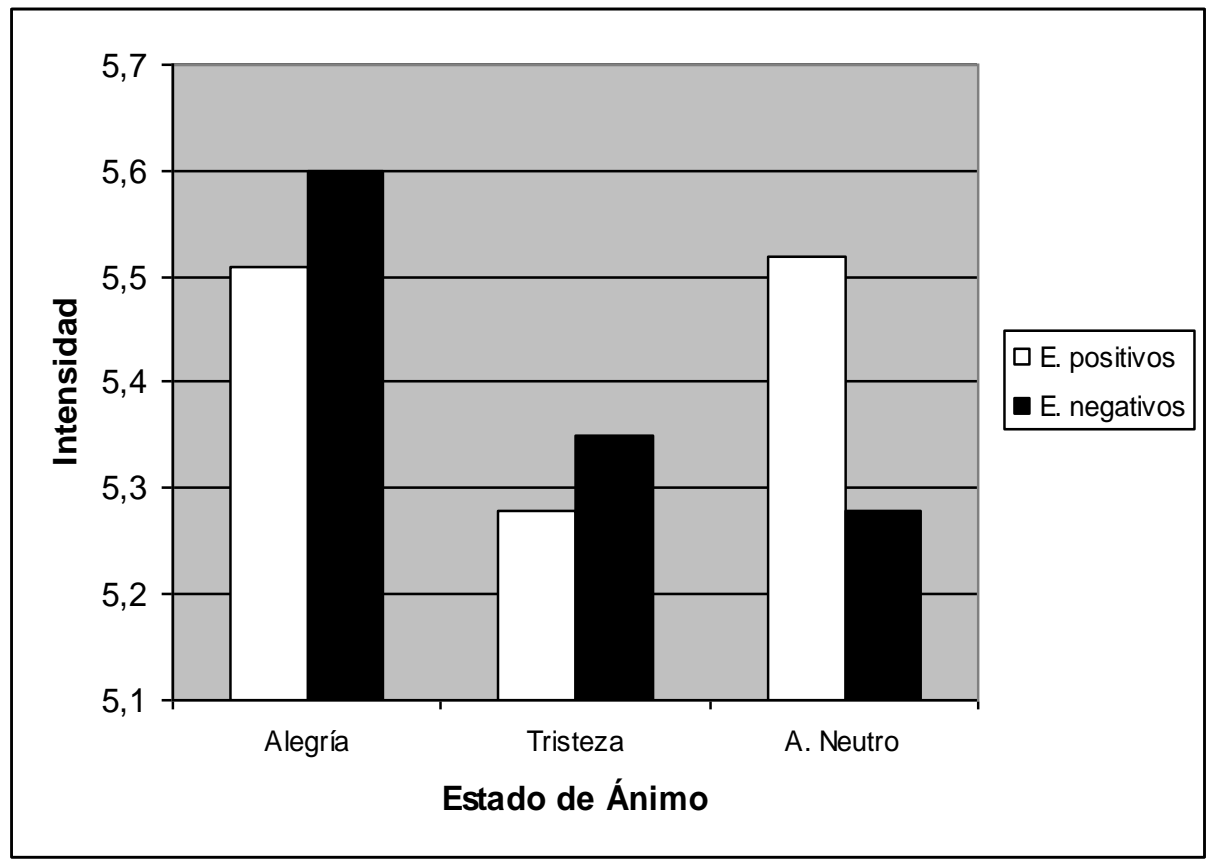

Figura 4. Medias de valoración de la intensidad en función del estado de ánimo y la valencia del evento.

Para analizar los efectos simples del estado de ánimo en cada nivel de valencia del evento, se ha realizado un ANOVA unifactorial. Los resultados muestran efectos significativos del estado de ánimo en la valoración de los eventos positivos $[F(2.66)=5.02$ y $p=.007]$. Los sujetos con estado de ánimo alegre o neutro han mostrado mayor valoración de intensidad de eventos positivos que los sujetos con ánimo triste $(M=5.51$ y $M=5.52$ vs. $M=5.28)$, como puede verse en la Figura 4. De igual modo, para el nivel de eventos negativos, el análisis ANOVA unifactorial $[F(2.66)=4.861$ у $p=$ .008] indica que los sujetos con ánimo alegre reportan una mayor intensidad emocional $(M=5.60$ vs. $M=5.27)$ que los sujetos con ánimo neutro.

Asimismo, se ha realizado un análisis de los efectos simples de la valencia del evento para cada nivel del factor estado de ánimo. Para los sujetos con estado de alegría, no hay diferencias significativas $[F(1.19)=1.21 \mathrm{y} p=.273]$ entre la valoración de eventos positivos $(M=5.51)$ y de los eventos negativos $(M=5.60)$. Para los sujetos con tristeza no hay diferencias significativas $[F(1.18)=0.55$ y $p=.458]$ en la valoración de la intensidad emocional de eventos positivos $(M=$ $5.28)$ y de los eventos negativos $(M=5.35)$. Sin embargo, el análisis de efectos simples de la valencia del evento para los sujetos con ánimo neutro $\left[F(1.18)=9.231\right.$ y $p=.003, \eta^{2}=$ $.048]$ indica que existen diferencias significativas. Los sujetos con ánimo neutro valoran los eventos positivos con mayor intensidad emocional que los eventos negativos $(M=5.2$ vs. $M=5.28)$.

\section{Discusión}

En primer lugar, hay que destacar que los resultados han mostrado un efecto de congruencia entre ánimo y memoria, pero el efecto es parcial. Se ha producido sólo en el plano de los recuerdos de valencia positiva. Los participantes alegres han valorado los recuerdos positivos con mayor intensidad que los participantes tristes. Por tanto, la confluencia de un ánimo positivo y una valencia positiva del material recuperado facilita el efecto MCM. Este resultado concuerda con el obtenido por Nouchi e Hyodo (2007). Estos autores, utilizando tareas autobiográficas, concluyeron que la valencia emocional del recuerdo recuperado influye en el efecto de congruencia. 
Sin embargo, se observa una asimetría, pues el efecto MCM no aparece si examinamos el plano de recuerdos negativos. En el grupo de tristeza, los participantes no valoraban los recuerdos negativos con intensidad superior al resto. Por tanto, se confirma que, a pesar de su robustez, el efecto de congruencia es más fiable en los estados de ánimo positivos (Blasco et al. 1997; Brewin, Andrews y Gotlib, 1993; Holland y Kensiguer, 2010; Rusting, 1998, 1999; Singer y Salovey, 1988). En una tarea de laboratorio, Blasco et al. (1997) observaron un aumento diferencial del número de palabras positivas recordadas en el grupo de inducción positiva. En suma, la investigación confirma que la alegría inducida tiende a facilitar la recuperación de material positivo, mientras un nivel leve de tristeza inducida a menudo no facilita la recuperación simétrica de contenidos negativos (Hasher et al., 1986; Isen, 1985).

La asimetría positiva es también visible en el grupo de ánimo neutro. Los participantes del grupo de inducción neutra han valorado con mayor intensidad emocional los eventos positivos, mientras han atribuido menor intensidad a los negativos. Este perfil indicaría que en la población general predomina un estado de ánimo general, crónicamente activo, para favorecer contenidos positivos en una dirección optimista o hedónica. Este resultado concuerda con estudios previos (Dreisbach y Goschke, 2004; Fiedler et al., 2001; Meilán et al., 2012). Si tenemos en cuenta este estado crónico de positividad presente en individuos sanos, podemos explicar la asimetría positiva frecuentemente asociada al efecto de congruencia.

Otro tipo de pruebas contribuyen a apoyar la hipótesis. El procesamiento positivo de fondo también se observa en estudios de neuroimagen (Deldin et al., 2001), en forma de sesgos observables cuando se trata de mantener información positiva. Análogamente, al someter a los participantes en un experimento a una tarea que exige la simulación de episodios futuros (D'Argembeau y Van der Linden, 2007; Gallo et al., 2011), se registra un número mayor de detalles contextuales o sensoriales cuando se trata de imaginar eventos positivos. Esta asimetría positiva en el procesamiento podría contribuir a la autorregulación emocional, el bienestar y la eficacia social.

En segundo lugar, hay que señalar en nuestros resultados las diferencias entre el grupo de alegría y el grupo tristeza en la valoración de los recuerdos -globalmente considerados-. Los participantes con estado de ánimo alegre valoran los eventos con mayor intensidad -tanto los positivos como los negativos-, respecto a los sujetos con ánimo triste, que valoran los eventos con una intensidad significativamente inferior. Esto ocurre sea cual sea el contexto en que se presente el material de inducción emocional.

Podemos encontrar una explicación en el modelo del afecto como información, de Storbeck y Clore (2008). Esta hipótesis supone que la activación emocional incrementa la dedicación a cualquier estrategia activa de procesamiento, influyendo en los juicios y valoraciones. Por consiguiente, el estado de ánimo influiría en la misma dirección sobre la valo- ración de los recuerdos positivos y negativos. En la misma línea, para Levine y Pizarro (2006) la emoción genera un estilo de procesamiento adaptativo a la situación. Según Fredrikson (1998, 2001, 2009), las emociones positivas generan un estilo de procesamiento más creativo, liberal y relacional, mientras las emociones negativas - cuando los objetivos están en riesgo- conducen a un estilo preferentemente conservador.

Estos resultados favorecen una concepción de los procesos emocionales como vectores organizadores o tendencias para la acción (Frijda, 1987), que influencian la cognición, incluyendo los procesos de recuperación (Teasdale y Barnard, 1993). La valoración más intensa de todos los sucesos relevantes de la historia escolar-incluidos los negativos-, bajo el influjo del ánimo positivo, es explicada por la intervención de procesos heurísticos más rápidos (Tiedens y Linton, 2001) y liberales (Kensinguer y Schacter, 2006), frente a estrategias de búsqueda más lentas (Storbeck y Clore, 2005), más sistemáticas y cuidadosas, bajo ánimo negativo (Levine y Bluck, 2004; Levine y Edelstein, 2009). El ánimo feliz intensificaría las evaluaciones, mientras el ánimo negativo llevaría a juicios más conservadores y estrictos. Este estilo de procesamiento afectaría en la misma dirección, para intensificar o disminuir, los eventos positivos o negativos. Por ejemplo, Alonso-Quecuty (1990) encuentra un patrón de simetría en la valencia de los recuerdos recuperados por sujetos con ánimo alegre o triste, después de un procedimiento de inducción. Este efecto del ánimo sobre el procesamiento cognitivo podría explicar las dificultades para observar el efecto MCM en determinados estudios (Blaney, 1986; Hasher et al., 1985).

En la misma línea, esta valoración de la intensidad emocional de un evento pasado en función del ánimo es congruente con la postura de Forgas (1995). Forgas propone que la influencia del afecto es mayor en tareas que exigen procesamiento más libre y creativo. Por tanto, la valoración de la intensidad sería dependiente de la estrategia cognitiva de procesamiento, en el caso de juicios personales. Explicaciones similares han sido propuestas por Fernández-Castro et al. (1997), que sugieren que el ánimo genera un tipo de procesamiento que dependería del contexto cognitivo más que de la experiencia emocional. Análogamente, Meilán et al. (2012) indican que los sesgos que produce el estado de ánimo en el procesamiento cognitivo podrían predisponer al sujeto para responder de forma heurística a estímulos relacionados con este estado emocional.

En cuanto a las limitaciones encontradas en este trabajo, se derivan de la incorporación de estímulos complejos y el uso de recuerdos personales. Estas características permiten incrementar la validez ecológica, pero originan otro tipo de dificultades. Por ejemplo, existe una limitación ética en los procesos de inducción afectiva, por lo que experimentalmente se producen estados de ánimo más débiles y difusos que en la vida real. Para superar este problema, podría recurrirse en futuras investigaciones al uso de emociones surgidas en situaciones naturales (ubicar la tarea de recuperación el mo- 
mento de recibir una calificación de examen). Así, las emociones naturales intensas permitirán comprobar si los recuerdos positivos se valoran más en estados positivos y si la tristeza intensifica de forma simétrica el material negativo.

Otra limitación puede estar relacionada con los efectos autorregulatorios. Nuestros resultados sugieren que los sujetos tienden a incrementar en la misma dirección los recuerdos positivos y negativos. Este efecto puede interpretarse como un mecanismo homeostático, compensatorio, de forma que se equilibran la valencia negativa y positiva para mantener el punto de equilibrio y evitar fluctuaciones anímicas que interferirían en otras tareas en curso. Para aislar las valoraciones de la influencia autorregulatoria y de la tendencia homeostática, la variable valencia del evento intrasujeto podría en el futuro ser manipulada a nivel intersujeto.

El tamaño del efecto ha sido pequeño, lo que refleja que sólo una pequeña parte de la variabilidad podría depender del estado de ánimo. Estos resultados indicarían que existe una estabilidad en las valoraciones de eventos relevantes de la historia vital, fruto de sucesivos procesos de repaso. Esta consistencia permitiría a la memoria autobiográfica servir a las funciones relacionadas con la identidad y la eficacia social. Además, en gran medida, los procesos reconstructivos y valorativos podrían depender de otras variables, relacionadas con rasgos de personalidad (Feldman-Barrett, 1997), con el nivel de auto-estima (Robinson y Clore, 2002), o con la habilidad para reducir el impacto emocional de eventos negativos (Fernández Berrocal y Extremera, 2006; Petrides y Furnham, 2003; Walker, Skowronski y Thompson, 2003). Explorar los efectos moderadores de estas variables puede resultar interesante en el futuro, debido a la relación de la memoria autobiográfica con la autorregulación emocional, el autoconcepto y el bienestar general.

Se podrían destacar algunas implicaciones educativas. En el área de la educación emocional, debe considerarse la superación de la dicotomía entre emociones positivas y negativas. Emociones como la tristeza, la nostalgia o la ansiedad, cuando se producen de forma transitoria y leve en individuos sanos, pueden ser adaptativas, puesto que ayudan a focalizar la atención en determinados aspectos de una situación.

En los programas de prevención dirigidos a adolescentes es importante tener en cuenta que no es necesario provocar estados emocionales intensos de euforia, fuera de lo normal. El estado emocional neutro que predomina durante la acti-

\section{Referencias}

Alonso-Quecuty, M.L. (1990). Memoria autobiográfica: la influencia de los estados de ánimo sobre las perspectivas del recuerdo. Estudios de Psicologia, 5-18, 43-44.

Blasco, T., Borràs, F.X., Rey, M., Bonillo, A. y Fernández Castro, J. (1997). Efectos de un procedimiento de inducción de estados de ánimo sobre el recuerdo de palabras. Anales de Psicología, 13, 163-176

Barry, E.S., Naus, M.J. y Rehm, L.P. (2004). Depression and implicit memory: Understanding mood congruent memory bias. Cognitive Therapy and Research, 28, 387-414. vidad cotidiana ya permite seleccionar una mayor proporción de contenidos positivos, así como otorgarles mayor peso o intensidad con la finalidad de favorecer la autoestima y la visión positiva de uno mismo.

Se podría apuntar una última repercusión en el ámbito clínico. En caso de trastorno esta asimetría positiva podría no producirse. Isen (1985) sugiere que los fracasos para observar los efectos de la depresión leve en el recuerdo no deben llevar a generalizar la conclusión de que los estados afectivos distintos de la tristeza ligera tienen efectos insignificantes en los procesos cognitivos. La confluencia del ánimo triste con contenidos autobiográficos negativos podría incidir en un descenso del nivel de autoestima o del bienestar. De este modo, los estados de tristeza intensos y estables llevarían a modificar el estilo cognitivo, generando sesgos para seleccionar contenidos negativos que agravarían el problema inicial. Si este ciclo se cronifica, aumentaría la probabilidad de sufrir un trastorno del estado de ánimo y este efecto de congruencia, simétrico en el plano negativo, podría ser visto como un índice útil para detectar una posible vulnerabilidad.

En suma, el efecto de congruencia se ha mostrado parcialmente y de forma asimétrica, ya que sólo se aprecia en la valencia positiva. El estado de tristeza inducida no parece incrementar la valoración de contenidos negativos. Esto no implica que no produzca un efecto cognitivo, sino que los efectos se traducen en una valoración más moderada y cuidadosa. Por tanto el perfil asimétrico positivo estaría asociado a la población general y podría considerarse un recurso del individuo dirigido a la autoprotección y la mejora del autoconcepto.

Visto en conjunto, el experimento muestra que una explicación a la asimetría del efecto de memoria congruente puede hallarse en la influencia específica de cada estado afectivo sobre la forma de procesamiento cognitivo. El estado afectivo selecciona el tipo de procesamiento más adecuado a la situación. Los estados de alegría tienden a producir valoraciones más intensas y juicios más expansivos, mientras la tristeza promueve valoraciones más ajustadas o sistemáticas. En síntesis, la interpretación de la emoción como información sobre los aspectos más relevantes de una situación puede ayudar a resolver las aparentes contradicciones o anomalías observadas en los experimentos sobre MCM. Los efectos del ánimo sobre la recuperación de recuerdos autobiográficos estarían modulados por el tipo de procesamiento cognitivo generado.

Blanchard, J. J., Bellack, A. S. y Mueser, K. T. (1994). Affective and socialbehavioral correlates of physical and social anhedonia in schizophrenia. Journal of Abnormal Psychology, 103, 719-728.

Blaney, P.H. (1986). Affect and memory: A review. Psychological Bulletin, 99, 229-246.

Bower, G. H. (1981). Mood and memory. American Psychologist, 36, 129-148.

Bower, G. H. (1987). Commentary on mood and memory. Behaviour Research and Therapy, 25, 443-455. 
Brewin, C.R., Andrews, B. y Gotlib, I.H. (1993). Psychopathology and early experience: A reappraisal of retrospective reports. Psychological Bulletin, 113, 82-98.

Christianson, S.A. (1992). Emotional stress and eyewitness memory: A critical review. Psychological Bulletin, 112, 284-309.

Conway, M.A. y Pleydell-Pearce, C. W. (2000). The construction of autobiographical memories in the self-memory system. Psychological Review, 107, 261-288

Ellis, H. C. y Moore, B. A. (1999). Mood and memory. En T. Dalgleish y M. J. Power (eds.), Handbook of cognition and emotion (pp. 193- 210). New York, NY: Wiley.

Ellis, H. C. y Ashbrook, P. W. (1991). The "state" of mood and memory research: A selective review. En D. Kuiken (ed.), Mood and Memory: Theory, Research, and Applications (pp. 185-196). Newbury Park, CA: Sage Publications.

Dreisbach, G. y Goschke, T. (2004). How positive affect modulates cognitive control: Reduced perseveration at the cost of increased distractibility. Journal of Experimental Psychology: Learning, Memory, and Cognition, 30, 343-353.

Deldin, P. J., Deveney, C. M., Kim, A. S., Casas, B. R. y Best, J. L. (2001). A slow wave investigation of working memory biases in mood disorders. Journal of Abnormal Psychology, 110, 267-281

D’Argembeau, A. y Van der Linden, M. (2007). Emotional aspects of mental time travel. Behavioral and Brain Sciences, 30, 220-221.

Feldman-Barrett, L. (1997). The relationships among momentary emotion experiences, personality descriptions, and retrospective ratings of emotion. Personality and Social Psychology Bulletin, 23, 1100-1110.

Fernández-Berrocal, P. y Extremera, N. (2006). Emotional intelligence and emotional reactivity and recovery in laboratory context. Psicothema, 18, $72-78$

Fernández-Castro, J., Granero, R., Barrantes, N. y Capdevila, A. (1997). Estados de ánimo y sesgos en el recuerdo: papel del afecto. Psicothema, 9, 247-258

Fredrickson, B. L. (2001). The role of positive emotions in positive psychology: The broaden and-build theory of positive emotions. American Psychologist, 56, 218-226.

Fredrickson, B. L. (2009). Positivity: Groundbreaking research reveals bow to embrace the bidden strength of positive emotions, overcome negativity, and thrive. New York, NY: Crown.

Fredrickson, B. L. (1998). What good are positive emotions? Review of General Psychology, 2, 300-319.

Frijda, N. H. (1987). Emotion, cognitive structure, and action tendency. Cognition and Emotion, 1, 115-143.

Fiedler, K., Nickel, S., Muehlfriedel, T. y Unkelbach, C. (2001). Is mood congruency an effect of genuine memory or response bias? Journal of Experimental social psychology, 37, 201-214.

Forgas, J. P. (1995). Mood and judgment: The affect infusion model (AIM). Psychological Bulletin, 117, 39-66.

Gallo, D. A, Korthauer, L. E., McDonough, I. M, Teshale, S. y Johnson, E. L. (2011). Age-related positivity effects and autobiographical memory detail: Evidence from a past/future source memory task. Memory, 19, 641-652.

Garcez, J. y Cláudio, V. (2009). Congruência de humor em memórias autobiográficas de infância de individuos com depressão. Análise Psicológica, 2, 159-173.

Gebauer, J., Broemer, P., Haddock, G. y von Hecker, U. (2008). Inclusionexclusion of positive and negative past selves: Mood congruence as information. Journal of Personality and Social Psychology, 95, 470-487.

Hasher, L., Rose, K.C., Zacks, R.T., Sanft, H. y Doren, B. (1985). Mood, recall, and selectivity in normal college students. Journal of Experimental Psychology: General, 114, 104-118.

Holland, A.C. y Kensinger, E.A. (2010). Emotion and autobiographical memory. Physics of Life Review, 7, 88-131.

Isen, A. M. (1985). Asymmetry of happiness and sadness in effects on memory in normal college students: Comment on Hasher, Rose, Zacks, Sanft, and Doren. Journal of Experimental Psychology: General, 114, 388391

Jakobs, E., Manstead, A. S. R. y Fischer, A. H. (2001). Social context effects on facial activity in a negative emotional setting. Emotion, 1, 51-69.
Kensinger, E.A. y Schacter, D.L. (2006). Processing emotional pictures and words: Effects of valence and arousal. Cognitive, Affective, and Behavioral Neuroscience, 6, 110-127.

Kreibig, S. D. (2004). Situational and Individual Response Specificity to Emotional Films.Design. Tesis, Universidad de Kiel. Recuperado de http://www.affective-sciences.org

Levine, L. J. (1997). Reconstructing memory for emotions. Journal of Experimental Psychology: General, 126, 165-177.

Levine, L. J. y Bluck, S. (1997). Experienced and remembered emotional intensity in older adults. Psychology and Aging, 12, 514-523.

Levine, L. J. y Bluck, S. (2004). Painting with broad strokes: Happiness and the malleability of event memory. Cognition and Emotion, 18, 559-574.

Levine, L. J. y Pizarro, D. A. (2006). Emotional valence, discrete emotions, and memory. En B. Uttl, N. Ohta y A. L. Siegenthaler (eds.), Memory and emotion: Interdisciplinary perspectives (pp. 37-58). Malden, MA: Blackwell Publishing.

Levine, L.J. y Edelstein, R S. (2009). Emotion and memory narrowing: A review and goal-relevance approach. Cognition \& Emotion, 23, 833- 875

Macht, M., Roth, S. y Ellgring, H. (2002).Chocolate eating in healthy men during experimentally induced sadness and joy. Appetite, 39, 147-158.

Madigan, R.J. y Bollenbach, A.K. (1982). Effects of induced mood on retrieval of personal episodic and semantic memories. Psychological Reports, 50, 147-157.

Mathews, A. y Bradley, B. (1983). Mood and the self-reference bias in recall. Behavior Research and Therapy, 21, 233-239.

Matt, G. E., Vázquez, C. y Campbell, W. K. (1992). Mood-congruent recall of affectively toned stimuli: A meta-analytic review. Clinical Psychology Review, 12, 227-255

Meilán, J. J., Carro, J., Guerrero, C., Carpi, A., Gómez, C., Palmero, F. (2012). El efecto de memoria congruente con el estado afectivo: reconocimiento diferencial de palabras de tristeza y alegría. Anales de Psi cología, 1, 266-273

Miranda, R. y Kihlstrom, J.F. (2005). Mood congruence in childhood and recent autobiographical memory. Cognition and Emotion, 19, 981-998.

Nouchi, R. y Hyodo, M. (2007). The congruence between the emotional valences of recalled episodes and mood states influences the mood congruence effect. Shinrigaku Kenkyu, 78, 25-32.

McHugo, G. J., Smith, C. A. y Lanzetta, J. T. (1982). The structure of selfreports of emotional responses to film segments. Motivation and Emotion 4, 365-385.

Petrides, K.V. y Furnham, A. (2003). Trait emotional intelligence: behavioural validation in two studies of emotion recognition and reactivity to mood induction. European Journal of Personality, 17, 39-57.

Philippot, P. (1993). Inducing and assessing differentiated emotional feeling states in the laboratory. Cognition and Emotion, 7, 171-193.

Robinson, M.D. y Clore, G.L. (2002). Belief and Feeling: Evidence for an accessibility model of emotional self-report. Psychological Bulletin, 128, 934-960.

Rubin, D.C., Burt, C. D. B. y Fifield, S. J. (2003). Experimental manipulations of the phenomenology of memory. Memory \& Cognition, 31, 877 886.

Rusting, C.L. (1998). Personality, mood, and cognitive processing of emotional information: Three conceptual frameworks. Psychological Bulletin 124, 165-196.

Rusting, C.L. (1999). Interactive effects of personality and mood on emotion-congruent memory and judgment. Journal of Personality and Social Psychology, 77, 1073-1086.

Schroots, J.J.F. (1996). Theoretical development in the Psychology of aging. The Gerontologist, 36, 742-748.

Schroots, J.J.F. y Assink, M.H.J. (2005). Portraits of life: Patterns of events over the lifespan. Journal of Adult Development, 12, 183-198.

Singer, J.A. y Salovey, P. (1988). Mood and memory: Evaluating the network theory of affect. Clinical Psychology Review, 8, 211-251.

Snyder, M. y White, P. (1982). Moods and memories: Elation, depression, and the remembering of the events of one's life. Journal of Personality, 50 , 142-167.

Storbeck, J. y Clore, G. L. (2008). Affective arousal as information: How af fective arousal influences judgments, learning, and memory. Social and Personality Psychology Compass, 2, 1824-1843. 
Talarico, J.M., LaBar, K.S. y Rubin, D.C. (2004). Emotional intensity predicts autobiographical memory experience. Memory \& Cognition, 32, $1118-1132$.

Teasdale, J.D. y Barnard, P.J. (1993). Affect, cognition, and change: re-modeling depressive thought. Hillsdale, NJ: Lawrance Erlbaum Associates.

Tiedens, L. Z. y Linton, S. (2001). Judgment under Emotional Certainty and Uncertainty: The Effects of Specific Emotions on Information Processing. Journal of Personality and Social Psychology, 81, 973-988.
Tulving, E. (1989). Memory: Performance, knowledge and experience. European Journal of Cognitive Psychology, 26, 1-12.

Walker, W. R., Skowronski J. J. y Thompson C. P. (2003). Life is pleasantand memory helps to keep it that way! Review of General Psychology, 7, 203 210.

Watson, D., Clark, L. A. y Tellegen, A. (1988). Development and validation of brief measures of positive and negative affect: The PANAS Scales. Journal of Personality and Social Psychology, 47, 1063-1070.

(Artículo recibido: 04-07-2012; revisado: 11-10-2014; aceptado: 19-10-2014) 